\title{
Predictive Maintenance Supported BY IIOT TECHNOLOGIES
}

\author{
Zeljko Stojkic, Igor Bosnjak, Luka Saravanja \& Eva Culjak
}
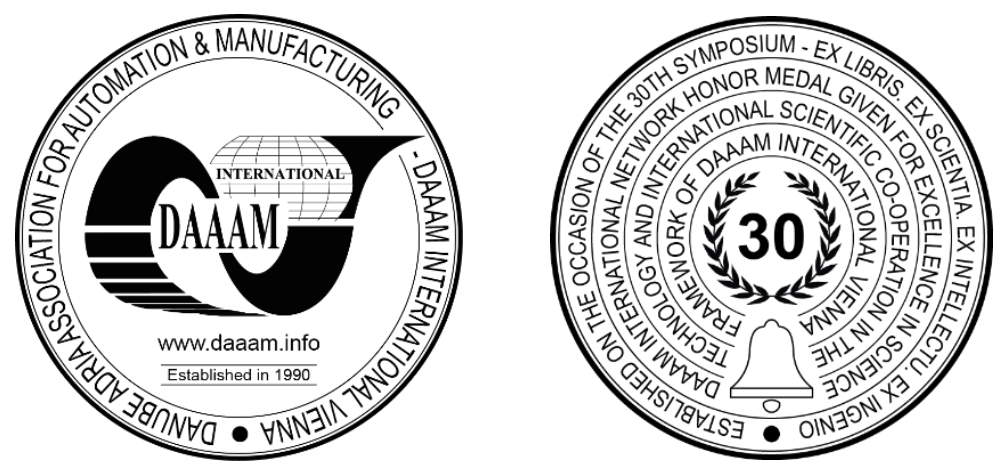

This Publication has to be referred as: Stojkic, Z[eljko]; Bosnjak, I[gor]; Saravanja, L[uka] \& Culjak, E[va] (2021). Predictive Maintenance Supported by IIoT Technologies, Proceedings of the 32nd DAAAM International Symposium, pp.0356-0362, B. Katalinic (Ed.), Published by DAAAM International, ISBN 978-3-902734-33-4, ISSN 1726-9679, Vienna, Austria

DOI: $10.2507 / 32$ nd.daaam.proceedings.052

\begin{abstract}
This paper describes new predictive maintenance trends and techniques. By applying new technologies within Industry 4.0, such as the Internet of Things, i.e., by processing data from many sensors, it is possible to identify potential problems in production facilities much before a failure occurs. Therefore, the paper describes a model of predictive maintenance using Industrial Internet of Things. It shows the architecture of predictive maintenance and its implementation on the 3D printer at the Learning Factory, University of Mostar. In addition to these technologies, for the full functioning of predictive maintenance, tools for processing and analysis of collected data such as machine learning methods are needed. Therefore, the paper presents ready-made machine learning solution. The paper shows that with modern technologies, such as the Internet of Things and Big Data Analysis, it is possible to integrate automated systems with IT systems, to better control and manage them, especially in the field of maintenance. Also, the goal of this paper is to contribute to the development of modern solutions in the field of predictive maintenance, the implementation of which in companies is one of the basic foundations for sustainable progress on the path of Industry 4.0.
\end{abstract}

Keywords: Predictive maintenance; Internet of Things; Industrial Internet of Things; Machine Learning

\section{Introduction}

Today's requirements in production, such as shorter production times, smaller series of products and higher quality, while keeping costs as low as possible, represent a great challenge for managing and organizing production. In order to be able to meet these trends, it is necessary to provide reliable production capacities, i.e., production machines and equipment that will be able to meet the planned production at the required time. Therefore, maintenance plays a key role in achieving these goals. However, today maintenance is also undergoing a transformation, previous strategies such as corrective and preventive maintenance do not meet the needs and requirements of production on the way to Industry 4.0. Industry 4.0 proposes the predictive manufacturing in the future industry. The machines are connected as a collaborative community. 
Such evolution requires the utilization of advance prediction tools, so that data can be systematically processed into information that can explain the uncertainties and thereby make more "informed" decisions [1]. The application of Industry 4.0 technologies such as the Internet of Things (IoT) and Big Data Analysis and the development of machine learning methods have enabled the development of predictive maintenance. Collecting data from various production equipment using the Industrial Internet of Things (IIoT) platform is a major step in building a predictive maintenance system, simplifying the maintenance process at industrial plants. Predictive maintenance is the direct monitoring of the condition and performance of equipment during normal operation to reduce the likelihood of failures and thus reduce costs by reducing the frequency of maintenance activity, reducing unplanned failures, and eliminating unnecessary preventive maintenance. Specific types of predictive maintenance include:

- Vibration analysis: Vibration sensors can be used to detect deterioration in the performance of equipment such as pumps and motors,

- Infrared analysis: Infrared cameras are often used to detect unusually high temperature conditions,

- Acoustic analysis: Acoustic analysis is performed by sound or ultrasound tests, to find gas or liquid leaks,

- Oil analysis: Oil analysis determines equipment wear by measuring the number and size of particles.

Predictive maintenance is an important enabler of Industry 4.0 concept. It is based on Computerized Maintenance Management System (CMMS) concept that takes advantage of state-of-the-art technological innovations. CMMS coordinates all activities related to the availability, productivity, and maintainability of Cyber-Physical Systems (CPSs). CMMS functioning is a data-driven process based on condition monitoring. High degree of automation is another crucial characteristic of such systems. All steps of a computerized maintenance process happen without or with minimal human involvement. Such approach prevents or minimises effects of human mistakes. To implement it, complex CMMS are required, which work fully autonomously, and are able to collect, store and analyse data continuously [2]. Moreover, when processed and analysed, these data can bring out valuable information and knowledge from manufacturing process and system dynamics. By applying analytic approaches based on data, it is possible to find interpretive results for strategic decision-making, providing advantages such as, maintenance cost reduction, machine fault reduction, repair stop reduction, spare parts inventory reduction, spare part life increasing, increased production, improvement in operator safety, repair verification, overall profit among others [3].

The same data can also be used to make energy consumption more transparent, allowing for further potential cost savings. Together, these forecasting mechanisms provide benefits to industry in the form of increased productivity and efficiency. Data generated from large sensor installations and other IIoT endpoints can be the basis for analytics to help accurately plan maintenance, based on changing equipment conditions and related processes. This approach can be extremely valuable in modernizing maintenance, reducing downtime, and optimizing the use of critical capital equipment. It is also important to emphasize the Prognostic and Health Management concept (PHM) that is closely related to maintenance approaches such as predictive maintenance. PHM is an engineering process where algorithms are used to detect anomalies, diagnose faults, and predict Remaining Useful Lifetime (RUL). Although the main goal of PHM is to provide the health state and estimate the RUL of the components or equipment, also financial benefits such as operational and maintenance cost reductions and extended lifetime are achieved [4].

\section{IoT architecture for predictive maintenance}

The paper proposes an architecture for predictive maintenance (PdM) based on IIoT, with all included modules that make it functional and as such can be implemented in intelligent and predictive maintenance. The scheme of the developed concept is shown in Figure 1. IoT sensors are used to monitor and collect data in real time. The collected data are then modelled into a PdM model using statistical tools and software. The components of PdM will be briefly described below.

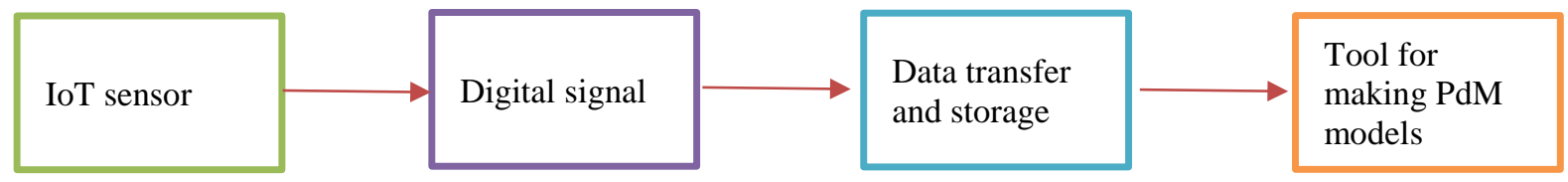

Fig. 1. IoT architecture for predictive maintenance

Sensor devices constitute at the same time the lowest level and the most important part of the system. In fact, they directly interact with the environment by measuring variables of interest and by processing acquired data to perform on board analysis tasks [5]. Few Popular Industrial IoT enabled sensors measure temperature, humidity, pressure, current, vibration, air quality, footfall, gas, and weight. Another commonly used sensor is the photoionization sensor, which is used to measure levels of volatile inorganic compounds and gases. The ultrasonic sound sensor is used to translate high frequency sounds due to leaks [6]. Data from sensors collected in the form of analogue signals are converted into a digital signal using an analogue digital converter. The digital signal is machine-readable and is used for further analysis. 
Digital data stream generated is stored on organizations' local intranet or a secure centralized or remote cloud location in the form of a database file. IoT sensors which are installed in remote locations without internet connectivity have inbuilt Random-Access Memory (RAM) and Storage space where data is stored. Data from IoT sensors is transmitted to a centralized cloud server or decentralized fog nodes via Wi-Fi, or in remote locations through Cellular networks or Bluetooth Low Energy (BLE) technology [6].

Machine Learning (ML), within artificial intelligence, has emerged as a powerful tool for developing intelligent predictive algorithms in many applications. ML approaches have the ability to handle high dimensional and multivariate data, and to extract hidden relationships within data in complex and dynamic environments (such as industrial environments) [3]. Based on the data collected and stored, it is possible to develop models and algorithms using machine learning that can help us predict future events based on past data. Machine learning means acquiring knowledge based on experience. It is not about remembering, but about finding rules in learning data. ML algorithms are categorized into three different types; supervised, unsupervised, and reinforcement learning (RL). The aim is to show how complex the structure can be and the commonly used available learning techniques. Moreover, different algorithms can be combined in order to maximize the classification power. To add on, some among the ML algorithms are both applicable to unsupervised and supervised learning [7].

\subsection{Selection of equipment to be applied for the case study at the Learning Factory}

For overview and presentation, the predictive maintenance architecture will be implemented on a 3D printer, inside the Learning Factory, from which data will be collected, i.e., temperature and vibrations. Based on the collected data, predictive analytics will be performed. By analysing the literature, possible solutions for the equipment needed for the realization of the definition of architecture were collected and systematized.

\begin{tabular}{|c|c|c|c|c|c|c|c|}
\hline $\begin{array}{c}\text { Architecture } \\
\text { level }\end{array}$ & \multicolumn{7}{|c|}{ Possible examples of a hardware solution } \\
\hline $\begin{array}{l}\text { Integration } \\
\text { into other } \\
\text { software }\end{array}$ & Grafana & Tableau & \multirow{4}{*}{$\begin{array}{l}\text { Siemens } \\
\text { Mindsphere }\end{array}$} & $\begin{array}{l}\text { Integration in } \\
\text { MES }\end{array}$ & \multirow{3}{*}{ Splunk } & $\begin{array}{l}\text { Production } \\
\text { applications }\end{array}$ & $\begin{array}{c}\text { PTC } \\
\text { ThingWorx } \\
\text { (Production } \\
\text { applications) }\end{array}$ \\
\hline Data analysis & & & & $\begin{array}{c}\text { Matlab } \\
\text { Statistics } \\
\end{array}$ & & \multirow{3}{*}{ Microsoft Azure } & PTC \\
\hline $\begin{array}{c}\text { Data } \\
\text { aggregation }\end{array}$ & Rapidminer & Python, R & & NODE-RED & & & $\begin{array}{l}\text { ThingWorx } \\
\text { (IIoT platform) }\end{array}$ \\
\hline Data storing & $\begin{array}{l}\text { Relational } \\
\text { database }\end{array}$ & $\begin{array}{l}\text { Hadoop } \\
\text { Cluster }\end{array}$ & & $\begin{array}{l}\text { Bosch IoT } \\
\text { Cloud }\end{array}$ & Edge server & & $\begin{array}{c}\text { PTC } \\
\text { ThingWorx } \\
\text { (KEPServerEX) }\end{array}$ \\
\hline $\begin{array}{c}\text { Protocol } \\
\text { (data) }\end{array}$ & MQTT & AMQP & OPC-UA & TCP/IP & LoRaWAN & HTTP & Modbus \\
\hline $\begin{array}{c}\text { Network } \\
\text { (Data) }\end{array}$ & LAN & LAN & $\begin{array}{c}\text { BLE } \\
\text { (Bluetooth) }\end{array}$ & $5 \mathrm{G}$ & NFC & 6LOWPAN & ZigBEE \\
\hline $\begin{array}{c}\text { Preprocessor } \\
\text { software }\end{array}$ & Node-RED & RapidMiner & R i Python & $\begin{array}{c}\text { Siemens } \\
\text { Simatic } \\
\end{array}$ & \multirow{4}{*}{$\begin{array}{c}\text { Bosch } \\
\text { XDK, } \\
\text { Bosch } \\
\text { Connected } \\
\text { Industrial } \\
\text { Sensor } \\
\text { (CISS) }\end{array}$} & $\begin{array}{l}\text { Microsoft Azura } \\
\text { IoT-Edge }\end{array}$ & $\begin{array}{c}\text { PTC } \\
\text { ThingWorx }\end{array}$ \\
\hline $\begin{array}{c}\text { Preprocessor } \\
\text { hardware }\end{array}$ & S7 1200 & $\begin{array}{l}\text { Harting } \\
\text { Mica }\end{array}$ & $\begin{array}{l}\text { Balluf IO } \\
\text { Link }\end{array}$ & $\begin{array}{l}\text { MindCOnnect } \\
2040 \\
\end{array}$ & & \multirow[b]{2}{*}{ PLC } & $\cdots$ \\
\hline $\begin{array}{c}\text { Interface for } \\
\text { embedded } \\
\text { systems }\end{array}$ & S7 1200 & $\mathrm{PC}$ & CAN-Bus & SPI-Bus & & & RS-232 \\
\hline Sensors & $\begin{array}{c}\begin{array}{c}\text { Siemens } \\
\text { sensors }\end{array} \\
\end{array}$ & $\begin{array}{c}\text { External } \\
\text { sensors }\end{array}$ & $\begin{array}{l}\text { Internal } \\
\text { sensors }\end{array}$ & $\begin{array}{c}\text { Bosch } \\
\text { Phantom }\end{array}$ & & $\cdots$ & $\cdots$ \\
\hline
\end{tabular}

Table 1. Possible examples of hardware solution by levels of architecture [4], [5], [6], [7]

Control unit

- Power supply for PLC, display and sensor, PM 190W

- Siemens S7-1200 PLC

Sensors

- Temperature transmitter AQZ-025, input Pt100, output 4-20mA, 24VDC

- Probe type Pt100, Ø3 x $50 \mathrm{~mm}, \mathrm{I}=1 \mathrm{~m}, 0-200^{\circ} \mathrm{C}$

- Humidity sensor with measuring transducer B + B Thermo-Technik

- Vibration monitoring module

- Vibration measuring sensor 

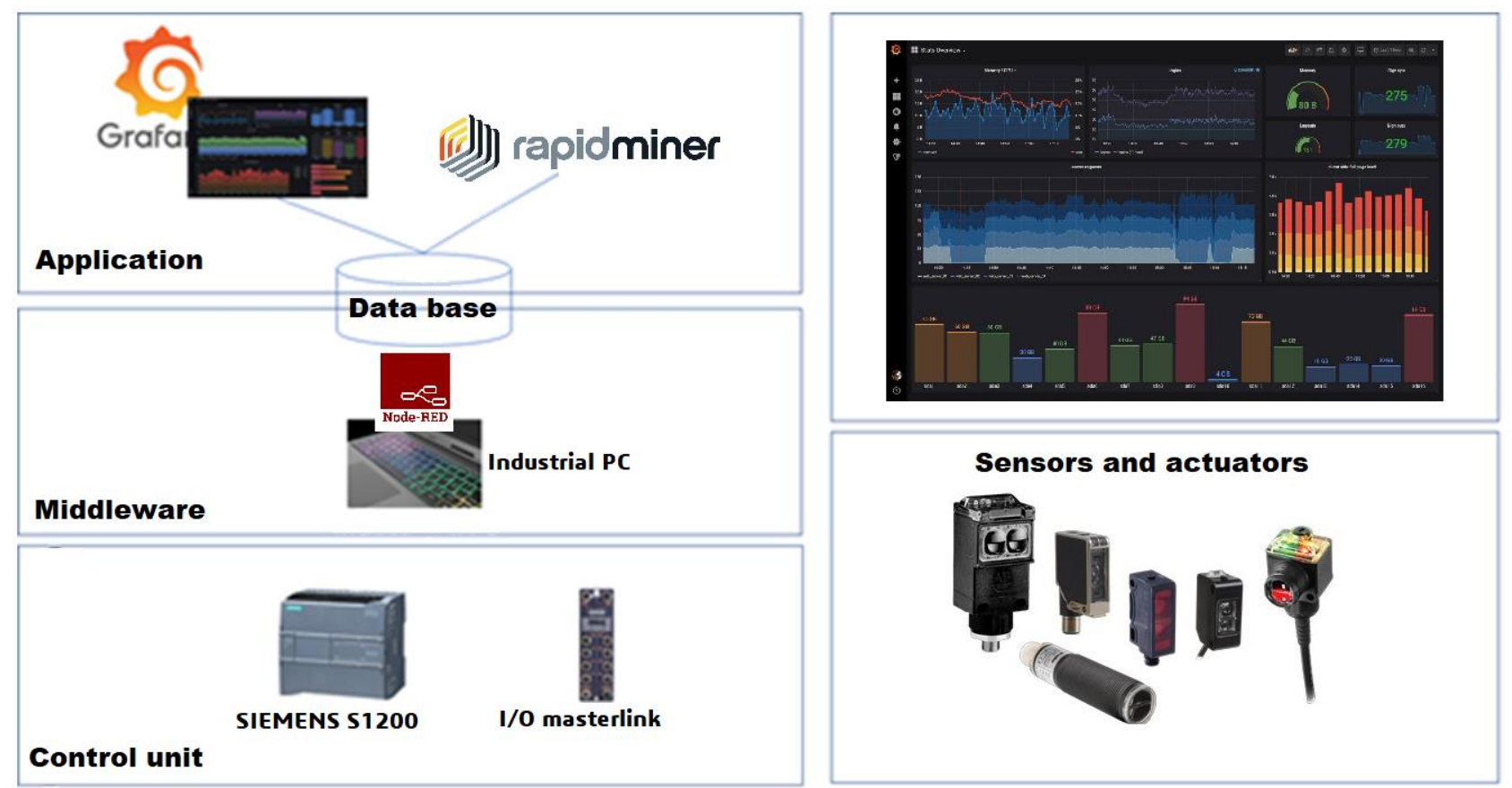

Fig. 2. Selected equipment architecture

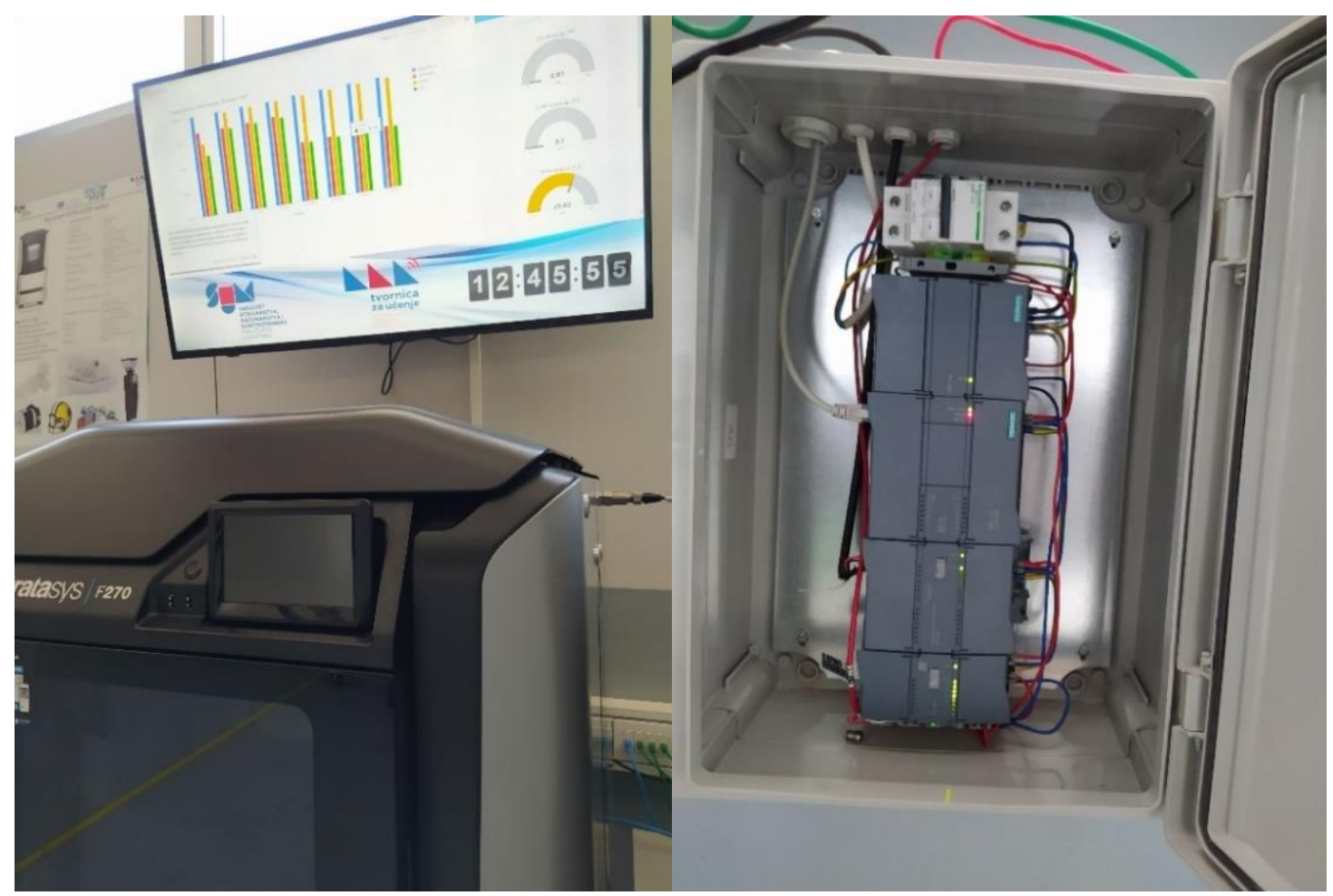

Fig. 3. a) The screen presents the data collected from the 3D printer, b) a cabinet with the entire control unit

\subsection{Middleware - connection between Siemens PLC and database}

An industrial computer is a standard computer that is used to install the necessary software for Node-RED and connect data from sensors via Siemens PLC via Node-RED and MQTT protocol to the database.

Node-RED is a flow-based development tool for visual programming developed originally by IBM for wiring together hardware devices, APIs, and online services as part of the Internet of Things [8]. Node-RED offers a webbased flow editor that can be used to create JavaScript functions. Application elements can be saved or shared for reuse. Runtime is built on Node.js. Streams created in Node-RED are stored using JSON. Node-Red in our architecture is used to connect between control hardware and the database. 


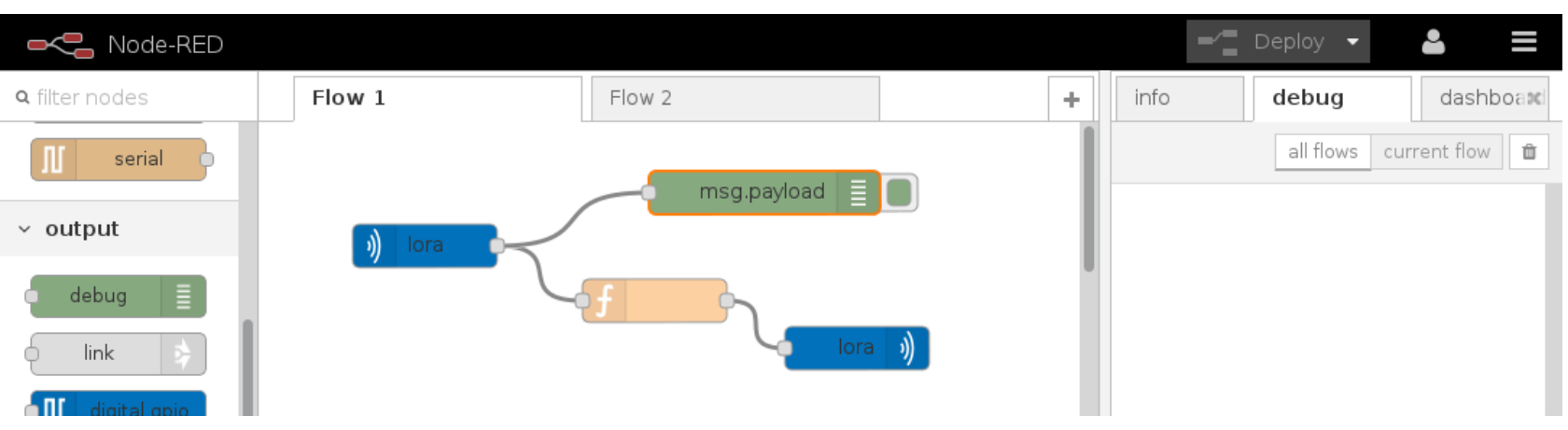

Fig. 4. Display the Node-RED tool interface

The MQTT protocol is an open, simple and lightweight protocol that allows distributed messaging between end nodes on a publish / subscribe basis. These features make it ideal for use in limited environments and low-bandwidth networks with limited processing capabilities, low memory capacity, and high latency. Messages are published on a specific channel through which the message is forwarded to all subscribers of that channel. The MQTT broker (MQTT broker) is in charge of comparing posts and subscriptions, which forwards published messages and remembers client subscriptions.

A node for Siemens PLC is installed within the Node-RED interface to allow access to data from the PLC. Each connection to the PLC is represented by the endpoint configuration node S7. We can configure the PLC address, the available variables and their addresses, and the variable read cycle time. Node-RED allows us to save data from nodes to the desired database, thus ensuring the periodic storage of data read from the sensor to the database.

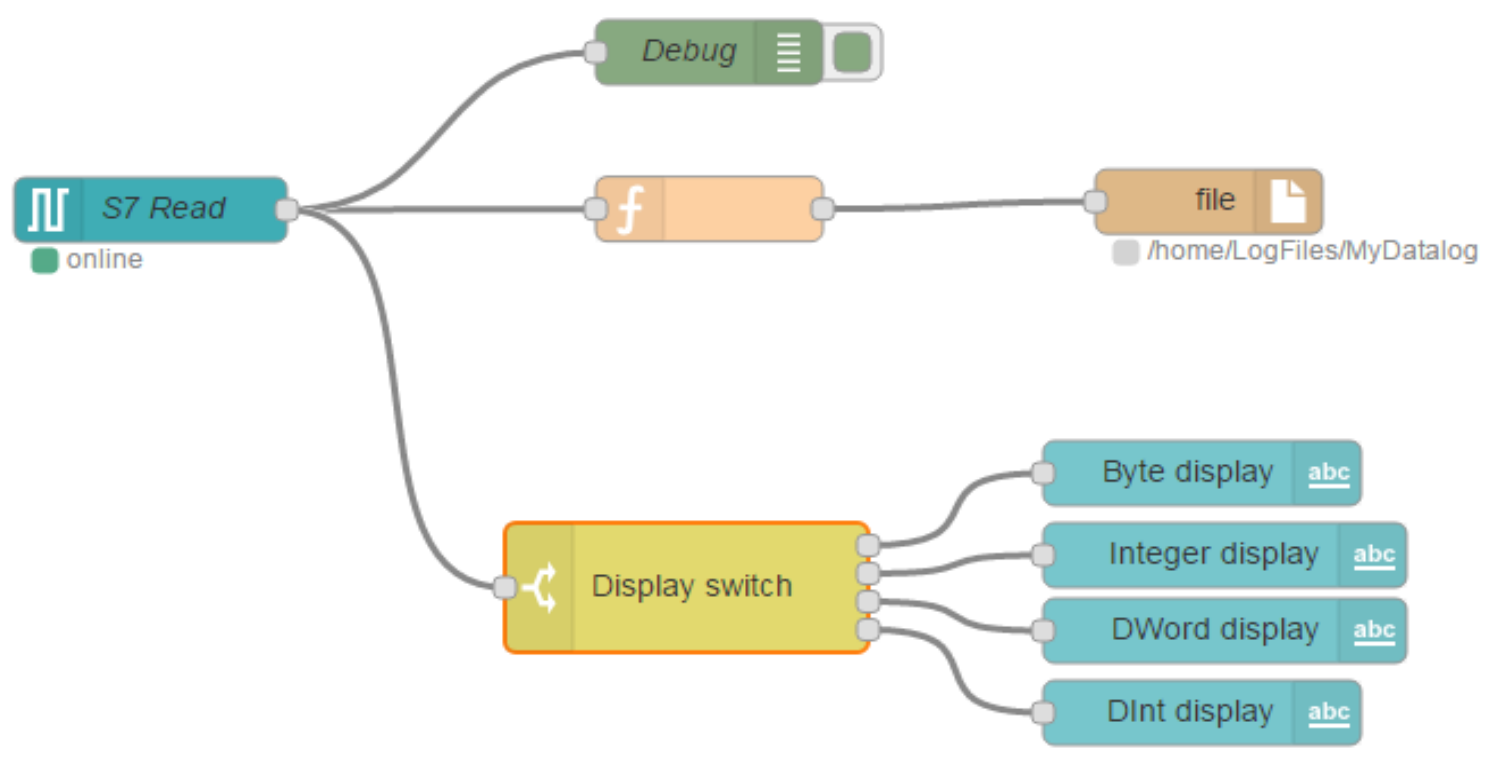

Fig. 5. Integration of Siemens PLC into Node-RED

\subsection{Tools for processing the collected data and presentation of predictive maintenance models}

It is planned to use the RapidMiner software tool, which integrates different machine learning methods, to create a predictive maintenance model. In the time of writing, we still did not develop a predictive maintenance model, which should process and analyse the data collected in the RapidMiner. But in the coming period, we plan to complete the model and describe it in future papers. Also, it is planned to use the Grafana software tool, to graphically present the obtained data and results. Below are briefly described software packages.

\subsubsection{RapidMiner - a software package for machine learning}

RapidMiner (RM) is a software platform for scientific data processing developed by the company of the same name. Provides an integrated environment for data preparation, machine learning, deep learning, text mining and predictive analytics. 
It is used for business and commercial applications, as well as for research, education, training, rapid prototyping and application development, and supports all steps of the machine learning process, including data preparation, visualization of results, validation and model optimization [9]. RapidMiner is an environment for machine learning and data mining processes. It is open-source, free project implemented in Java. It represents a new approach to design even very complicated problems - a modular operator concept which allows the design of complex nested operator chains for a huge number of learning problems. RM uses XML to describe the operator trees modelling knowledge discovery (KD) processes. RM has flexible operators for data input and output in different file formats. It contains more than 100 learning schemes for regression, classification, and clustering tasks [10].

The work in the software itself requires experience and prior preparation of the collected data, which must be adjusted before the analysis, i.e., perform the necessary data operations, which include data cleaning, data collection, data sorting and data formatting. After preparing the data, machine learning operations can be performed, in order to obtain a predictive model These operations are performed to draw out meaning information from data in order to make better decisions towards business. Analytics can be performed with various machine learning or custom algorithmic concepts like regression, classification, clustering, model-based recommendation etc. These models are further evaluated and improved by various stages of machine learning concepts. Improved or optimized algorithms can provide better results [11].

After creating the model, it needs to be visualized. RM provides a high level of visualization support. It is possible within the software to create detailed results of data analyses. In addition, the visualization of nodes and other information can be highly colourful and appealing to the eye. In this way, RapidMiner can allow users with higher level programming and coding skills to have increased output from their efforts in terms of being able to visualize the data and results. However, without at least some basic programming and coding skills, it seems unlikely that the full performance of the visualization abilities of the software could be achieved. The reason for this is that the visualization support within the software is connected to the functions that are performed [12].

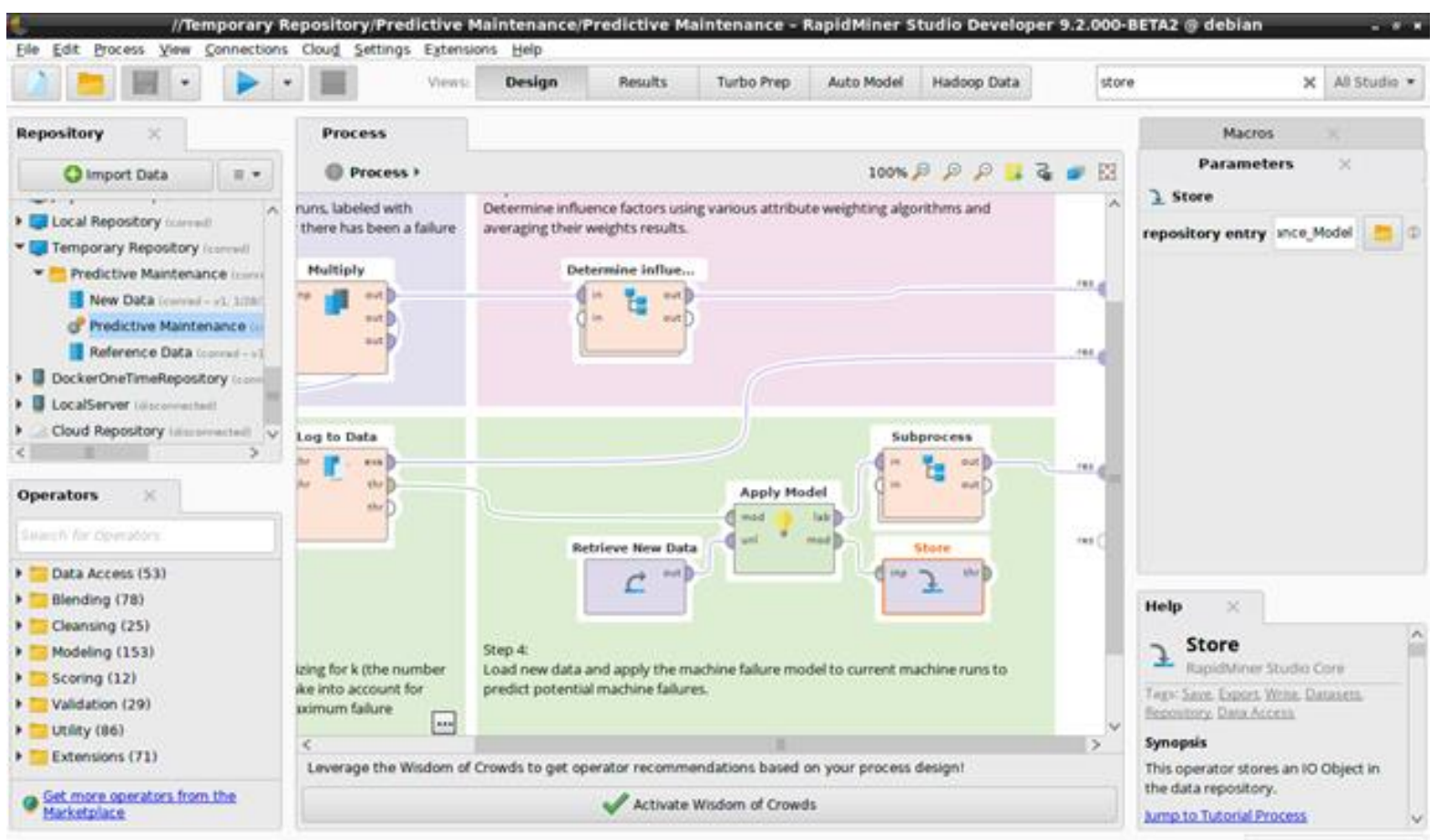

Fig. 6. RapidMiner software tool interface

\subsubsection{Grafana - software package for visualization and display of data}

Grafana is a multiplatform open-source analytical and interactive visualization web tool. Allows you to create charts, graphs, and alerts for the web when connected to supported data sources. It is extensible via a plugin system. End users can create complex dashboards using interactive query tools.

\section{Conclusion}

Finally, we can conclude that in today's conditions and requirements in production, predictive maintenance of production plants and machines plays an important role in the sustainability and profitability of the company's business. The development and application of new technologies in industry, i.e., the application of IIoT, has enabled the development and implementation of predictive systems in maintenance. 
With such systems, cost savings can be expected, as there should be no sudden breakdowns and unnecessary preventive maintenance activities. One of the problems the companies encounter in migrating to IIoT is the fact that different devices have used different protocols to send and receive data to each other in the past. Although several different communication protocols are currently used, due to their low cost and security in communication, the MQTT protocol is the standard for IIoT. In order to remain competitive, companies are committed to modernizing systems and equipment to meet new regulations and keep pace with growing market demands and stability. Companies that have embraced IIoT have made significant improvements in safety, efficiency, and profitability, and this trend is expected to continue with the spread and adoption of new IIoT technologies. Also, in order to complete the architecture of the predictive model, customized solutions in the field of artificial intelligence such as machine learning methods are needed, which will be able to accept and process the collected data and make decisions in the maintenance and production function. One of the limitations of such a model is the need for machine learning experts, who will be able to develop and optimize prediction models in a ready-made tool such as RapidMiner. To date, a large number of algorithms have been developed in the field of machine learning, but with the constant development of new digital technologies, algorithms often need to be adapted and optimized. Therefore, future work will be based on further development of the architecture of the predictive maintenance model, which will include the use of new solutions in IIoT and the development and optimization of algorithms for predicting and forecasting the condition of individual machines on the basis of which faster and more accurate decisions will be made regarding the reliability and condition of individual plants and machines. Also, future works will monitor the implementation of such systems in production facilities.

\section{References}

[1] Deac, C. N.; Deac, C. D.; Popa, C. L.; Ghinea, M \& Cotet, E. C. (2017). Using augmented reality in smart manufacturing, Proceedings of the 28th DAAAM International Symposiums, pp. 0727-0732, B. Katalinic (Ed.), Published by DAAAM International, ISBN 978-3-902734-11-2, ISSN 1726-9679, Vienna, Austria DOI: 10.2507/28th.daaam.proceedings. 102

[2] Katona, A \& Panfilov, P (2018). Building predictive maintenance framework for smart environment application systems, Proceedings of the 29th DAAAM International Symposium, pp.0460-0470, B. Katalinic (Ed.), Published by DAAAM International, ISBN 978-3-902734-20-4, ISSN 1726-9679, Vienna, Austria DOI: 10.2507/29th.daaam.proceedings.068

[3] Carvalho, T. P. et al. (2019). A systematic literature review of machine learning methods applied to predictive maintenance. Computers \& Industrial Engineering Vol. 137, November 2019, 106024, DOI: 10.1016/j.cie.2019.106024

[4] Cachada et al. (2018). Maintenance 4.0: intelligent and predictive maintenance system architecture, Proceedings of 23rd International Conference on Emerging Technologies and Factory Automation (ETFA), ISBN 978-1-53867109-2, ISSN 1946-0740, DOI: 10.1109/ETFA.2018.8502489

[5] Civerchia, F.; Bocchino, S.; Salvadori, C.; Rossi, E.; Maggiani, L. \& Petracca, M. (2017). Industrial internet of things monitoring solution for advanced predictive maintenance applications. Journal of Industrial Information Integration Vol. 7, September 2017, Pages 4-12 DOI: 10.1016/j.jii.2017.02.003

[6] Nangia, S.; Makkar, S. \& Hassan R. (2020). IoT based predictive maintenance in manufacturing sector, Proceedings of the International Conference on Innovative Computing \& Communications (ICICC) 2020, DOI: $10.2139 /$ ssrn.3563559

[7] Cinar, Z. M. et al. (2020). Machine learning in predictive maintenance towards sustainable smart manufacturing in industry 4.0. Sustainability Vol. 12, October 2020, DOI: 10.3390/su12198211

[8] Heath, N. (2014). "How IBM's Node-RED is hacking together the internet of things". techrepublic.com. CBS Interactive. Retrieved January 16, 2017

[9] Hofmann, M. \& Klinkenberg, R. (2013). RapidMiner: data mining use cases and business analytics application, CRC Press, ISBN 9781482205497

[10] Graczyk, M.; Lasota, T. \& Trawinski, B. (2009) Comparative analysis of premises valuation models using KEEL, RapidMiner and WEKA, Proceedings of the International Conference on Computational Collective Intelligence 2009, Wroclaw Poland, DOI: 10.1007/978-3-642-04441-0_70

[11] Dwivedi, S.; kasliwal, P. \& Soni, S. (2016). Comprehensive study of data analytics tool (RapidMIner, Weka, R tool, Knime), Symposium on Colossal Data Analysis and Networking (CDAN) Indore India, ISBN 978-1-50900670-0 DOI: 10.1109/CDAN.2016.7570894

[12] Hirudkar, A. M. \& Sherekar, S. S. (2013). Comparative analysis of data mining tools and techniques for evaluating of database systems, International Journal Of Computer Science And Applications Vol. 6, No.2, April 2013, ISSN: 0974-1011 